\title{
A sufficient condition for reciprocal Jacobi triangles
}

\author{
Sandra Lieven ${ }^{1}$ \\ Colégio Albert Sabin, São Paulo, SP \\ Márcio Fabiano da Silva² \\ Centro de Matemática, Computação e Cognição, UFABC, Santo André, SP
}

\begin{abstract}
Given a triangle $A B C$ let $A^{\prime} B^{\prime} C^{\prime}$ be a Jacobi triangle for $A B C$. When $\angle B A^{\prime} C^{\prime}=$ $\angle C A^{\prime} B^{\prime}=\alpha^{\prime}, \angle A B^{\prime} C^{\prime}=\angle C B^{\prime} A^{\prime}=\beta^{\prime}$ and $\angle A C^{\prime} B^{\prime}=\angle B C^{\prime} A^{\prime}=\gamma^{\prime}$, the triangle $A B C$ is a Jacobi triangle for $A^{\prime} B^{\prime} C^{\prime}$. In this case we say that $A B C$ and $A^{\prime} B^{\prime} C^{\prime}$ are reciprocal Jacobi triangles. In 2015 , G.T. Vickers presented a necessary condition for two triangles to be reciprocal, but the question whether that condition was also sufficient remained open. In this work we prove it by using basically trigonometric relations.
\end{abstract}

Keywords. Jacobi's Theorem, Triangle, Geometry.

\section{Introduction}

On the sides of a given (arbitrary) triangle $A B C$ construct three similar isosceles triangles $A B C^{\prime}, B C A^{\prime}, A C B^{\prime}$, exterior to $A B C$, such that the angular measure $\angle B A C^{\prime}=\angle A B C^{\prime}=$ $\angle B C A^{\prime}=\angle C B A^{\prime}=\angle A C B^{\prime}=\angle C A B^{\prime}$. Ludwig Kiepert showed that in this case the lines $\overleftrightarrow{A A^{\prime}}$, $\overleftrightarrow{B B^{\prime}}$ and $\overleftrightarrow{C C^{\prime}}$ are concurrent at a point $K$, which is called the Kiepert point of the triangle $A B C$. Carl Friedrich Andreas Jacobi (1795-1855), a German mathematician who devoted himself to study the triangular geometry, generalized the Kiepert's construction as it follows:

Theorem 1.1. (Jacobi's theorem) Let $A B C$ be an arbitrary triangle, $A^{\prime}, B^{\prime}, C^{\prime}$ points such that $\angle C^{\prime} A B=\angle C A B^{\prime}=\alpha, \angle A^{\prime} B C=\angle C^{\prime} B A=\beta$ and $\angle B^{\prime} C A=\angle A^{\prime} C B=\gamma$. The lines $\overleftrightarrow{A A^{\prime}}, \overleftrightarrow{B B^{\prime}}$ and $\overleftrightarrow{C C^{\prime}}$ are concurrent at a point $K$.

The triangle $A^{\prime} B^{\prime} C^{\prime}$ and the point $K$ are called the Jacobi triangle for the triangle $A B C$ with respect to the angles $\alpha, \beta, \gamma$ and the Jacobi point for the triangle $A^{\prime} B^{\prime} C^{\prime}$, respectively. We illustrate one Jacobi triangle in Figure 1. We consider $\alpha, \beta, \gamma \in(0, \pi)$, but they can be naturally extended to the interval $(0,2 \pi)$.

There are many proofs for Theorem 1.1. One idea to prove the existence of $K$ is to apply the following important result of the geometry of triangles:

(Ceva's theorem) The three lines containing the vertices $A, B, C$ of the triangle $A B C$ and intersecting the opposite sides in points $P, Q$, and $R$, respectively, are concurrent if and only if $\frac{B R}{R A} \cdot \frac{C P}{B P} \cdot \frac{A Q}{Q C}=1$.

This strategy was used in [2] to prove Theorem 1.1. Here we present a scheme for it. The details can be viewed in [1].

\footnotetext{
${ }^{1}$ sandralieven@gmail.com

2 marcio.silva@ufabc.edu.br
} 


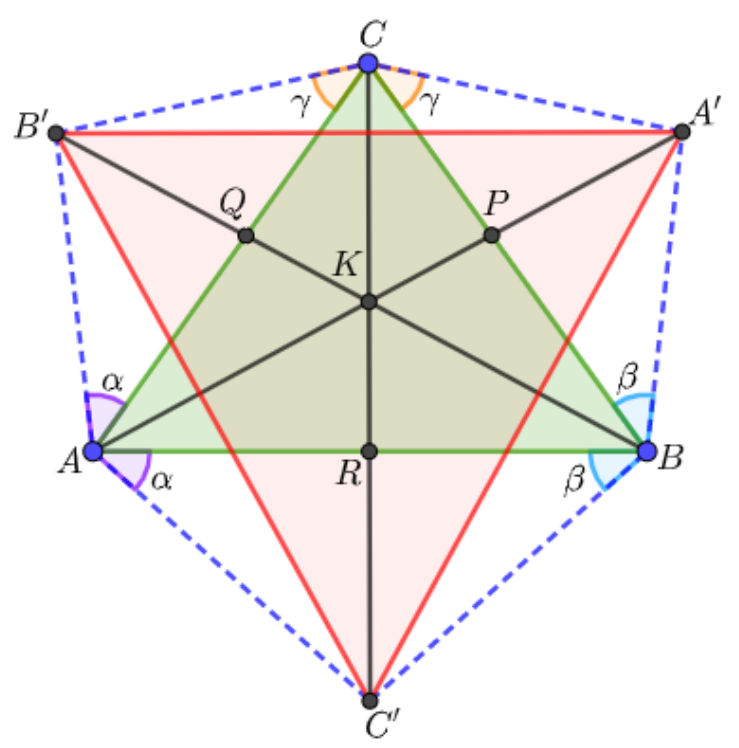

Figure 1: The Jacobi triangle $A^{\prime} B^{\prime} C^{\prime}$ for the triangle $A B C$.

Proof. As illustrated in Figure 1, let $\{P\}=\overleftrightarrow{A A^{\prime}} \cap \overleftrightarrow{B C},\{Q\}=\overleftrightarrow{B B^{\prime}} \cap \overleftrightarrow{A C}$ and $\{R\}=\overleftrightarrow{C C^{\prime}} \cap \overleftrightarrow{A B}$ In Figure 2 we see a schematic proof that $\frac{B R}{R A} \cdot \frac{C P}{B P} \cdot \frac{A Q}{Q C}=1$.

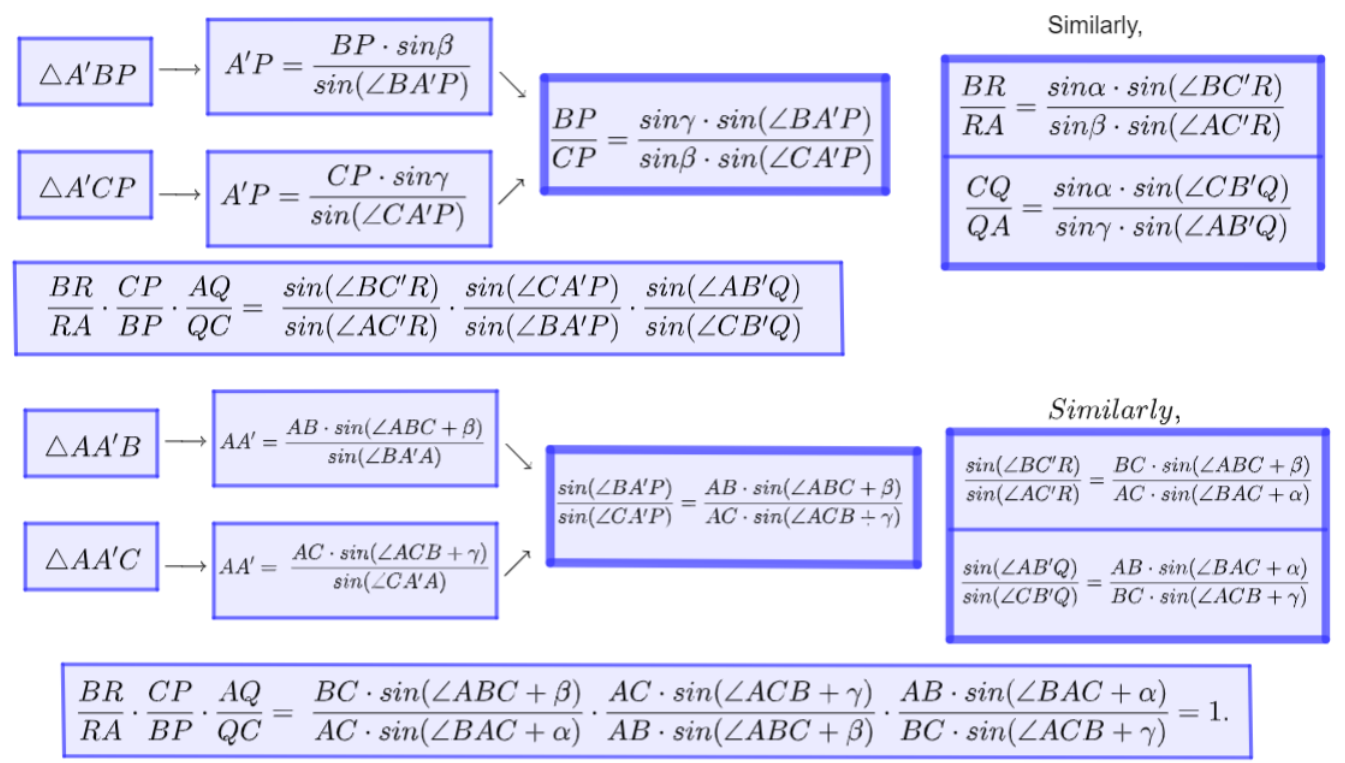

Figure 2: Scheme for Jacobi's theorem proof.

From Ceva's theorem it follows that $\overleftrightarrow{B B^{\prime}} \cap \overleftrightarrow{A A^{\prime}} \cap \overleftrightarrow{C C^{\prime}}=\{K\}$ 


\section{Reciprocal Jacobi triangles}

Definition 2.1. Let $A B C$ be any triangle and $A^{\prime} B^{\prime} C^{\prime}$ be the Jacobi triangle for $A B C$ with respect to the angles $\alpha, \beta, \gamma$ constructed on the vertices $A, B, C$, respectively. If the Jacobi triangle with angles $\alpha^{\prime}, \beta^{\prime}$ and $\gamma^{\prime}$ for the triangle $A^{\prime} B^{\prime} C^{\prime}$ coincides with $A B C$, we say that $A B C$ and $A^{\prime} B^{\prime} C^{\prime}$ are reciprocal Jacobi triangles.

In Figure 3 we can see two reciprocal Jacobi triangles.

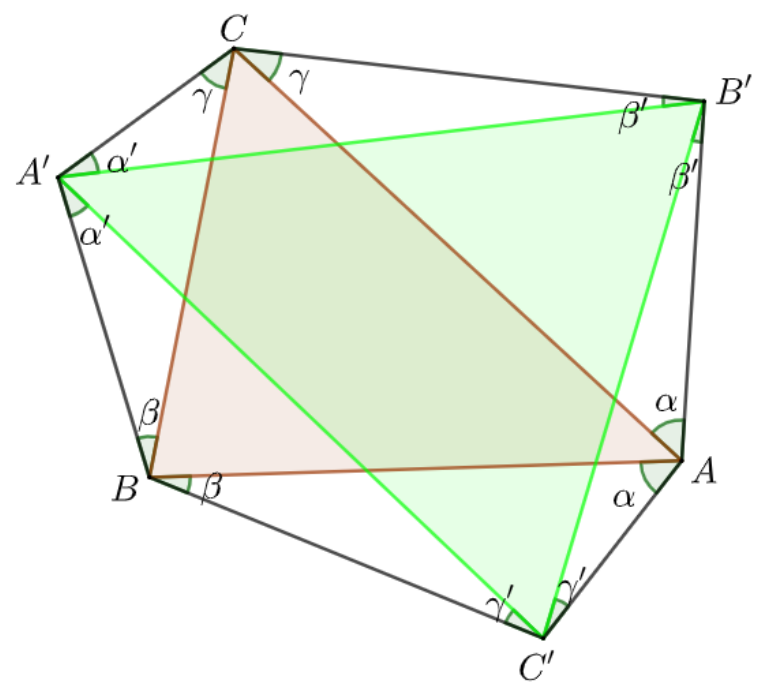

Figure 3: $A B C$ and $A^{\prime} B^{\prime} C^{\prime}$ are reciprocal Jacobi triangles.

In the conditions of Definition 2.1, Vickers has shown in [2] that if $A B C$ and $A^{\prime} B^{\prime} C^{\prime}$ are reciprocal Jacobi triangles then

$$
\frac{\sin (\angle B A C+2 \alpha)}{\sin (\angle B A C)}=\frac{\sin (\angle A B C+2 \beta)}{\sin (\angle A B C)}=\frac{\sin (\angle A C B+2 \gamma)}{\sin (\angle A C B)} .
$$

The converse of that result was an open question since 2015. We proved it during Sandra Lieven's master's studies and present it bellow.

Proposition 2.1. Let $A B C$ be any triangle and $A^{\prime} B^{\prime} C^{\prime}$ its Jacobi triangle with angles $\alpha, \beta, \gamma$ constructed on $A, B, C$, respectively, such that

$$
\frac{\sin (\angle B A C+2 \alpha)}{\sin (\angle B A C)}=\frac{\sin (\angle A B C+2 \beta)}{\sin (\angle A B C)}=\frac{\sin (\angle A C B+2 \gamma)}{\sin (\angle A C B)}=\mu,
$$

where $\mu$ is a real constant. Then $A B C$ and $A^{\prime} B^{\prime} C^{\prime}$ are reciprocal Jacobi triangles.

Proof. Let $\angle C A^{\prime} B^{\prime}=\alpha^{\prime}, \angle B A^{\prime} C^{\prime}=\theta, \angle A B^{\prime} C^{\prime}=\beta^{\prime}, \angle C B^{\prime} A^{\prime}=\phi, \angle B C^{\prime} A^{\prime}=\gamma^{\prime}$ and $\angle A C^{\prime} B^{\prime}=$ $\psi$, as illustrated in Figure 4.

By the law of sines for triangles $A B C^{\prime}$ and $A B^{\prime} C^{\prime}$ we have

$$
\frac{A C^{\prime}}{\sin \beta}=\frac{B C^{\prime}}{\sin \alpha}=\frac{A B}{\sin (\pi-\alpha-\beta)}=\frac{A B}{\sin (\alpha+\beta)} \quad \text { and } \quad \frac{A C^{\prime}}{\sin \beta^{\prime}}=\frac{A B^{\prime}}{\sin \psi} .
$$




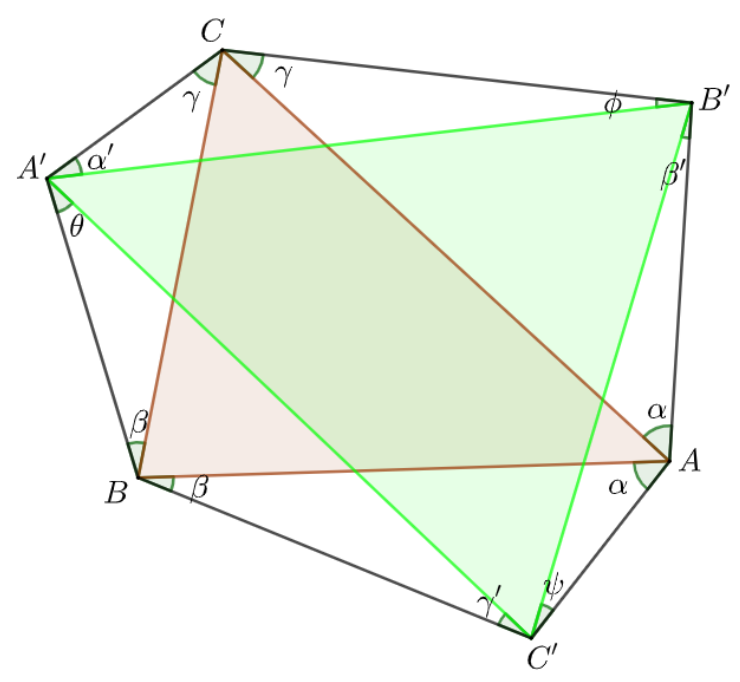

Figure 4: We need to prove that $\theta=\alpha^{\prime}, \phi=\beta^{\prime}$ and $\psi=\gamma^{\prime}$.

Consequently,

$$
\frac{A B \cdot \sin \beta}{\sin (\alpha+\beta)}=A C^{\prime}=\frac{A B^{\prime} \cdot \sin \beta^{\prime}}{\sin \psi},
$$

so that

$$
\frac{A B \cdot \sin \beta}{\sin (\alpha+\beta)} \cdot \frac{1}{\sin \beta^{\prime}}=\frac{A B^{\prime}}{\sin \psi} .
$$

From the law of sines for triangle $A B^{\prime} C$ it follows that

$$
A B^{\prime}=\frac{A C \cdot \sin \gamma}{\sin (\alpha+\gamma)}
$$

From (1) and (2) we get

$$
\frac{A B \cdot \sin \beta}{\sin (\alpha+\beta)} \cdot \frac{1}{\sin \beta^{\prime}}=\frac{A C \cdot \sin \gamma}{\sin (\alpha+\gamma)} \cdot \frac{1}{\sin \psi} .
$$

From triangle $A B^{\prime} C^{\prime}$ we have $\psi=\pi-\angle B A C-2 \alpha-\beta^{\prime}$. Then

$$
\begin{gathered}
\frac{A B \cdot \sin \beta}{\sin (\alpha+\beta)} \cdot \frac{1}{\sin \beta^{\prime}}=\frac{A C \cdot \sin \gamma}{\sin (\alpha+\gamma) \cdot \sin \left(\angle B A C+2 \alpha+\beta^{\prime}\right)} \Longrightarrow \\
\frac{\sin \left(\angle B A C+2 \alpha+\beta^{\prime}\right)}{\sin \beta^{\prime}}=\frac{A C \cdot \sin \gamma \cdot \sin (\alpha+\beta)}{A B \cdot \sin \beta \cdot \sin (\alpha+\gamma)} \Longrightarrow \\
\frac{\sin (\angle B A C+2 \alpha) \cdot \cos \beta^{\prime}+\sin \beta^{\prime} \cdot \cos (\angle B A C+2 \alpha)}{\sin \beta^{\prime}}=\frac{A C \cdot \sin \gamma \cdot \sin (\alpha+\beta)}{A B \cdot \sin \beta \cdot \sin (\alpha+\gamma)} \Longrightarrow \\
\sin (\angle B A C+2 \alpha) \cdot \cot \beta^{\prime}+\cos (\angle B A C+2 \alpha)=\frac{A C \cdot \sin \gamma \cdot \sin (\alpha+\beta)}{A B \cdot \sin \beta \cdot \sin (\alpha+\gamma)} \Longrightarrow \\
\cot \beta^{\prime}=\frac{A C \cdot \sin \gamma \cdot \sin (\alpha+\beta)}{A B \cdot \sin \beta \cdot \sin (\alpha+\gamma) \cdot \sin (\angle B A C+2 \alpha)}-\cot (\angle B A C+2 \alpha) .
\end{gathered}
$$


Since

$$
\frac{\sin (\angle B A C+2 \alpha)}{\sin (\angle B A C)}=\mu
$$

we obtain

$$
\mu \cdot \cot \beta^{\prime}=\frac{A C \cdot \sin \gamma \cdot \sin (\alpha+\beta)}{A B \cdot \sin \beta \cdot \sin (\alpha+\gamma) \cdot \sin (\angle B A C)}-\frac{\cos (\angle B A C+2 \alpha)}{\sin (\angle B A C)} .
$$

From triangle $A B C$,

$$
\frac{A C}{A B}=\frac{\sin (\angle A B C)}{\sin (\angle A C B)}
$$

and $\angle A B C=\pi-\angle A C B-\angle B A C$. Thus $\sin (\angle A B C)=\sin (\angle B A C+\angle A C B)$ and

$$
\begin{gathered}
\frac{A C}{A B}=\frac{\sin (\angle B A C+\angle A C B)}{\sin (\angle A C B)}=\frac{\sin (\angle B A C) \cdot \cos (\angle A C B)+\sin (\angle A C B) \cdot \cos (\angle B A C)}{\sin (\angle A C B)} \Longrightarrow \\
\frac{A C}{A B}=\sin (\angle B A C) \cdot \cot (\angle A C B)+\cos (\angle B A C) .
\end{gathered}
$$

From (3) and (4) we have that $\mu \cdot \cot \beta^{\prime}=$

$(\sin (\angle B A C) \cdot \cot (\angle A C B)+\cos (\angle B A C)) \cdot \frac{\sin \gamma \cdot \sin (\alpha+\beta)}{\sin \beta \cdot \sin (\alpha+\gamma) \cdot \sin (\angle B A C)}-\frac{\cos (\angle B A C+2 \alpha)}{\sin (\angle B A C)}$.

So

$$
\mu \cdot \cot \beta^{\prime}=(\cot (\angle A C B)+\cot (\angle B A C)) \cdot \frac{\sin \gamma \cdot \sin (\alpha+\beta)}{\sin \beta \cdot \sin (\alpha+\gamma)}-\frac{\cos (\angle B A C+2 \alpha)}{\sin (\angle B A C)} .
$$

But

$$
\begin{gathered}
-\frac{\cos (\angle B A C+2 \alpha)}{\sin (\angle B A C)}=\frac{-\cos (\angle B A C) \cdot \cos 2 \alpha+\sin (\angle B A C) \cdot \sin 2 \alpha}{\sin (\angle B A C)} \Longrightarrow \\
-\frac{\cos (\angle B A C+2 \alpha)}{\sin (\angle B A C)}=-\cot (\angle B A C) \cdot \cos 2 \alpha+\sin 2 \alpha \Longrightarrow \\
-\frac{\cos (\angle B A C+2 \alpha)}{\sin (\angle B A C)}=\frac{1}{\sin 2 \alpha} \cdot\left(\sin ^{2} 2 \alpha-\cot (\angle B A C) \cdot \cos 2 \alpha \cdot \sin 2 \alpha\right) \Longrightarrow \\
-\frac{\cos (\angle B A C+2 \alpha)}{\sin (\angle B A C)}=\frac{1}{\sin 2 \alpha} \cdot\left(1-\cos ^{2} 2 \alpha-\frac{\cos (\angle B A C)}{\sin (\angle B A C)} \cdot \cos 2 \alpha \cdot \sin 2 \alpha\right) \Longrightarrow \\
-\frac{\cos (\angle B A C+2 \alpha)}{\sin (\angle B A C)}=\frac{1}{\sin 2 \alpha} \cdot\left(1-\cos ^{2} 2 \alpha\left(\cos 2 \alpha+\sin 2 \alpha \cdot \frac{\cos (\angle B A C)}{\sin (\angle B A C)}\right)\right) \Longrightarrow \\
-\frac{\cos (\angle B A C+2 \alpha)}{\sin (\angle B A C)}=\frac{1}{\sin 2 \alpha} \cdot\left(1-\cos 2 \alpha\left(\frac{\sin (\angle B A C) \cdot \cos 2 \alpha+\sin 2 \alpha \cdot \cos (\angle B A C)}{\sin (\angle B A C)}\right)\right) .
\end{gathered}
$$

Thus

$$
-\frac{\cos (\angle B A C+2 \alpha)}{\sin (\angle B A C)}=\frac{1}{\sin 2 \alpha} \cdot(1-\cos 2 \alpha \cdot \mu)=-\frac{(\mu \cdot \cos 2 \alpha-1)}{\sin 2 \alpha} .
$$


By replacing (6) in (5) we get

$$
\mu \cdot \cot \beta^{\prime}=(\cot (\angle A C B)+\cot (\angle B A C)) \cdot \frac{\sin \gamma \cdot \sin (\alpha+\beta)}{\sin \beta \cdot \sin (\alpha+\gamma)}-\frac{(\mu \cdot \cos 2 \alpha-1)}{\sin 2 \alpha} .
$$

Analogously

$$
\mu \cdot \cot \phi=(\cot (\angle A C B)+\cot (\angle B A C)) \cdot \frac{\sin \alpha \cdot \sin (\gamma+\beta)}{\sin \beta \cdot \sin (\alpha+\gamma)}-\frac{(\mu \cdot \cos 2 \gamma-1)}{\sin 2 \gamma} .
$$

If $\alpha=\gamma$ then by (7) and (8) we have $\mu \cdot \cot \beta^{\prime}=\mu \cdot \cot \phi$. Therefore $\beta^{\prime}=\phi$ for $\beta^{\prime}, \phi \in(0, \pi)$.

We now suppose that $\alpha \neq \gamma$. Since

$\mu=\frac{\sin (\angle B A C+2 \alpha)}{\sin (\angle B A C)}=\frac{\sin (\angle B A C) \cdot \cos 2 \alpha+\sin 2 \alpha \cdot \cos (\angle B A C)}{\sin (\angle B A C)}=\cos 2 \alpha+\sin 2 \alpha \cdot \cot (\angle B A C)$, thus

$$
\cot (\angle B A C)=\frac{\mu}{\sin 2 \alpha}-\cot 2 \alpha
$$

In a similar way,

$$
\cot (\angle A C B)=\frac{\mu}{\sin 2 \gamma}-\cot 2 \gamma
$$

From (9) and (10) we get

$$
\begin{gathered}
\cot (\angle B A C)+\cot (\angle A C B)=\frac{\mu}{\sin 2 \alpha}-\cot 2 \alpha+\frac{\mu}{\sin 2 \gamma}-\cot 2 \gamma= \\
=\frac{\mu \cdot(\sin 2 \alpha+\sin 2 \gamma)-\cos 2 \alpha \cdot \sin 2 \gamma-\cos 2 \gamma \cdot \sin 2 \alpha}{\sin 2 \alpha \cdot \sin 2 \gamma}=\frac{\mu \cdot(\sin 2 \alpha+\sin 2 \gamma)-\sin (2 \alpha+2 \gamma)}{\sin 2 \alpha \cdot \sin 2 \gamma} .
\end{gathered}
$$

Thus

$$
\sin 2 \alpha \cdot \sin 2 \gamma \cdot(\cot (\angle B A C)+\cot (\angle A C B))=\mu \cdot(\sin 2 \alpha+\sin 2 \gamma)-\sin (2 \alpha+2 \gamma) .
$$

But $\cot \beta^{\prime}=\cot \phi \Longleftrightarrow \mu \cdot \cot \beta^{\prime}=\mu \cdot \cot \phi \Longleftrightarrow$

$$
\begin{gathered}
(\cot (\angle A C B)+\cot (\angle B A C)) \cdot \frac{\sin \gamma \cdot \sin (\alpha+\beta)}{\sin \beta \cdot \sin (\alpha+\gamma)}-\frac{(\mu \cdot \cos 2 \alpha-1)}{\sin 2 \alpha}= \\
=(\cot (\angle A C B)+\cot (\angle B A C)) \cdot \frac{\sin \alpha \cdot \sin (\gamma+\beta)}{\sin \beta \cdot \sin (\alpha+\gamma)}-\frac{(\mu \cdot \cos 2 \gamma-1)}{\sin 2 \gamma} \Longleftrightarrow \\
\frac{(\cot (\angle A C B)+\cot (\angle B A C))}{\sin \beta \cdot \sin (\alpha+\gamma)} \cdot(\sin \alpha \cdot \sin (\gamma+\beta)-\sin \gamma \cdot \sin (\alpha+\beta))= \\
=\frac{(\mu \cdot \cos 2 \gamma-1)}{\sin 2 \gamma}-\frac{(\mu \cdot \cos 2 \alpha-1)}{\sin 2 \alpha} \Longleftrightarrow \\
(\cot (\angle A C B)+\cot (\angle B A C)) \cdot \sin 2 \alpha \cdot \sin 2 \gamma \cdot\left(\frac{\sin \alpha \cdot \sin (\gamma+\beta)-\sin \gamma \cdot \sin (\alpha+\beta)}{\sin \beta \cdot \sin (\alpha+\gamma)}\right)= \\
=\mu(\cos 2 \gamma \cdot \sin 2 \alpha-\cos 2 \alpha \cdot \sin 2 \gamma)-\sin 2 \alpha+\sin 2 \gamma \Longleftrightarrow \\
(\cot (\angle A C B)+\cot (\angle B A C)) \cdot \sin 2 \alpha \cdot \sin 2 \gamma \cdot \\
\left(\frac{\sin \alpha \cdot \sin \beta \cdot \cos \gamma+\sin \alpha \cdot \sin \gamma \cdot \cos \beta-\sin \gamma \cdot \sin \alpha \cdot \cos \beta-\sin \gamma \cdot \sin \beta \cdot \cos \alpha}{\sin \beta \cdot \sin (\alpha+\gamma)}\right)=
\end{gathered}
$$




$$
\begin{gathered}
=\mu \cdot \sin (2 \alpha-2 \gamma)-2 \cdot \sin \left(\frac{2 \alpha-2 \gamma}{2}\right) \cdot \cos \left(\frac{2 \alpha+2 \gamma}{2}\right) \Longleftrightarrow \\
(\cot (\angle A C B)+\cot (\angle B A C)) \cdot \sin 2 \alpha \cdot \sin 2 \gamma \cdot \frac{\sin \beta \cdot(\sin \alpha \cdot \cos \gamma-\sin \gamma \cdot \cos \alpha)}{\sin \beta \cdot \sin (\alpha+\gamma)}= \\
=\mu \cdot \sin (2 \alpha-2 \gamma)-2 \cdot \sin (\alpha-\gamma) \cdot \cos (\alpha+\gamma) \Longleftrightarrow \\
(\cot (\angle A C B)+\cot (\angle B A C)) \cdot \sin 2 \alpha \cdot \sin 2 \gamma \cdot \frac{\sin (\alpha-\gamma)}{\sin (\alpha+\gamma)}= \\
=\mu \cdot 2 \sin (\alpha-\gamma) \cdot \cos (\alpha-\gamma)-2 \cdot \sin (\alpha-\gamma) \cdot \cos (\alpha+\gamma) .
\end{gathered}
$$

Since $\sin (\alpha-\gamma) \neq 0$ for $\alpha \neq \gamma$, we have that $\mu \cdot \cot \beta^{\prime}=\mu \cdot \cot \phi \Longleftrightarrow$

$$
\begin{gathered}
(\cot (\angle A C B)+\cot (\angle B A C)) \cdot \sin 2 \alpha \cdot \sin 2 \gamma= \\
=2 \mu \cdot \sin (\alpha+\gamma) \cdot \cos (\alpha-\gamma)-2 \cdot \sin (\alpha+\gamma) \cdot \cos (\alpha+\gamma) \Longleftrightarrow \\
(\cot (\angle A C B)+\cot (\angle B A C)) \cdot \sin 2 \alpha \cdot \sin 2 \gamma=2 \mu \cdot \sin (\alpha+\gamma) \cdot \cos (\alpha-\gamma)-\sin (2 \alpha+2 \gamma) \\
\Longleftrightarrow(\cot (\angle A C B)+\cot (\angle B A C)) \cdot \sin 2 \alpha \cdot \sin 2 \gamma= \\
=-\sin (2 \alpha+2 \gamma)+2 \mu \cdot(\sin \alpha \cdot \cos \gamma+\sin \gamma \cdot \cos \alpha) \cdot(\cos \alpha \cdot \cos \gamma+\sin \alpha \cdot \sin \gamma) \Longleftrightarrow \\
(\cot (\angle A C B)+\cot (\angle B A C)) \cdot \sin 2 \alpha \cdot \sin 2 \gamma= \\
=-\sin (2 \alpha+2 \gamma)+2 \mu \cdot\left(\sin \alpha \cdot \cos \alpha \cdot\left(\sin ^{2} \gamma+\cos ^{2} \gamma\right)+\cos \gamma \cdot \sin \gamma \cdot\left(\sin ^{2} \alpha+\cos ^{2} \alpha\right) .\right.
\end{gathered}
$$

Therefore $\mu \cdot \cot \beta^{\prime}=\mu \cdot \cot \phi \Longleftrightarrow$

$$
(\cot (\angle A C B)+\cot (\angle B A C)) \cdot \sin 2 \alpha \cdot \sin 2 \gamma=-\sin (2 \alpha+2 \gamma)+\mu \cdot(\sin 2 \alpha+\sin 2 \gamma),
$$

which is true by (11). We then get $\beta^{\prime}=\phi$ for $\beta^{\prime}, \phi \in(0, \pi)$.

Analogously we can conclude that $\alpha^{\prime}=\theta$ and $\gamma^{\prime}=\psi$ so that $A B C$ and $A^{\prime} B^{\prime} C^{\prime}$ are reciprocal Jacobi triangles.

\section{Conclusions}

Let $A B C$ an arbitray triangle and $A^{\prime} B^{\prime} C^{\prime}$ its Jacobi triangle with angles $\alpha, \beta, \gamma$ constructed on the vertices $A, B, C$, respectively, as it is illustrated in Figure 1 . We say that $A B C$ and $A^{\prime} B^{\prime} C^{\prime}$ are reciprocal Jacobi triangles if $A B C$ can be obtained from $A^{\prime} B^{\prime} C^{\prime}$ through a Jacobi construction, that is, if $\angle B A^{\prime} C^{\prime}=\angle C A^{\prime} B^{\prime}, \angle A B^{\prime} C^{\prime}=\angle C B^{\prime} A^{\prime}$ and $\angle A C^{\prime} B^{\prime}=\angle B C^{\prime} A^{\prime}$. It is possible to determine reciprocal Jacobi triangles by studying the following trigonometric condition

$$
\frac{\sin (\angle B A C+2 \alpha)}{\sin (\angle B A C)}=\frac{\sin (\angle A B C+2 \beta)}{\sin (\angle A B C)}=\frac{\sin (\angle A C B+2 \gamma)}{\sin (\angle A C B)}
$$

which involves only the internal angles of the triangle $A B C$ and $\alpha, \beta, \gamma$. In [2] it was shown that (12) is a necessary condition to $A B C$ and $A^{\prime} B^{\prime} C^{\prime}$ be reciprocal Jacobi triangles. In this work we proved that (12) is also a sufficient condition, which was an open question since 2015.

\section{References}

[1] Lieven, S. Triângulo de Jacobi, Dissertação de Mestrado, UFABC, 2019.

[2] Vickers, G. T. Reciprocal Jacobi Triangles and the McCay Cubic, Forum Geometricorum, 15: 179-183, 2015. 